\title{
An Outbreak of Ralstonia pickettii Bloodstream Infection Associated with an Intrinsically Contaminated Normal Saline Solution
}

\author{
Yin-Yin Chen, PhD; ${ }^{1}$ Wan-Tsuei Huang, MS; ${ }^{2}$ Chia-Ping Chen, BS; ${ }^{2}$ Shu-Mei Sun, MS; ${ }^{2}$ Fu-Mei Kuo, MS; ${ }^{3}$ \\ Yu-Jiun Chan, $\mathrm{PhD}{ }^{4}$ Shu-Chen Kuo, $\mathrm{MD} ;{ }^{5}$ Fu-Der Wang, $\mathrm{MD}^{6}$
}

ов јестіуе. Ralstonia pickettii has caused contamination of pharmaceutical solutions in many countries, resulting in healthcare infections or outbreak events. We determined the source of the outbreak of $R$. pickettii bloodstream infection (BSI).

Methods. This study was conducted in a 3,000-bed tertiary referral medical center in Taiwan with >8,500 admissions during May 2015. Patients had been treated in the injection room or chemotherapy room at outpatient departments, emergency department, or hospital wards. All patients who were culture positive for $R$. pickettii from May 3 to June 11, 2015, were eligible for the study. The aim of the survey was to conduct clinical epidemiological and microbiological investigations to identify possible sources of infection.

RESULTS. We collected 57 R. pickettii-positive specimens from 30 case patients. We performed 24 blood cultures; 14 of these revealed $>2$ specimens and 6 used fluid withdrawn from Port-a-Cath implantable venous access devices. All patients received an injection of $20 \mathrm{~mL} 0.9 \%$ normal saline via catheter flushing. In addition, 2 unopened ampules of normal saline solution ( $20 \mathrm{~mL})$ were confirmed positive for $R$. pickettii. The Taiwan Centers for Disease Control and Prevention performed sampling and testing of the same manufactured batch and identified the same strain of $R$. pickettii. Pulsed-field gel electrophoresis tests revealed that all clinical isolates had similarity of $>90 \%$, validating the outbreak of the same clone of $R$. pickettii.

CONCLUSIONS. R. pickettii can grow in saline solutions and cause bloodstream infections. Hospital monitoring mechanisms are extremely important measures in identifying and ending such outbreaks.

Infect Control Hosp Epidemiol 2017;38:444-448

Bloodstream infection (BSI) outbreaks due to contamination of pharmaceutical solutions during manufacturing occur rarely. ${ }^{1,2}$ Most nosocomial outbreaks of infusion-related BSIs have been derived from contamination of infusate by gram-negative bacilli (GNB) introduced during manufacturing. Among these microorganisms, Ralstonia pickettii has been reported to cause infection via contaminated solutions. ${ }^{3}$ Solutions contaminated at the point of manufacture have also been associated with outbreaks of BSIs or catheter-related infections. ${ }^{4,5}$

The R. pickettii bacterium is waterborne and can survive and grow in various water sources, including water supplies, sterile water, intravenous ranitidine, narcotics, skin disinfectants, blood culture bottles, saline solutions, magnesium vials, and other solutions used for patient care in hospitals. ${ }^{4,6-10}$ Those contaminated solutions can be administered by vascular injection, by intravenous drip infusion, or via wound cleaning, resulting in BSIs or other infections. Numerous additional outbreaks caused by $R$. pickettii contamination of saline solution have been reported by hospitals in several countries, ${ }^{2}$ and most of these contamination events occurred during the manufacturing stage. ${ }^{11}$

Although the pathogenicity of this bacterium is not strong, it has higher virulence for immunosuppressed patients and those who are debilitated in some way. ${ }^{12-14}$

In our hospital, a total of 4 blood specimens from 4 patients were culture positive for $R$. pickettii within 1 week. We conducted clinical epidemiological and microbiological investigations to identify possible sources of infection and to block the route of infection to end the outbreak.

\footnotetext{
Affiliations: 1. Department of Infection Control and Department of Nursing, Taipei Veterans General Hospital; National Yang-Ming University, Taipei, Taiwan; 2. Department of Infection Control and Department of Nursing, Taipei Veterans General Hospital, Taipei, Taiwan; 3. Department of Infection Control and Department of Pathology and Laboratory Medicine, Taipei Veterans General Hospital, Taipei, Taiwan; 4. Department of Medicine, and Department of Pathology and Laboratory Medicine, Taipei Veterans General Hospital; National Yang-Ming University, Taipei, Taiwan; 5. National institute of Infectious Diseases and Vaccinology, National Health Research Institutes; 6. Division of Infectious Diseases, Department of Infection Control, and Department of Medicine, Taipei Veterans General Hospital; National Yang-Ming University, Taipei, Taiwan.

Received September 21, 2016; accepted December 1, 2016; electronically published Januray 24, 2017

(c) 2017 by The Society for Healthcare Epidemiology of America. This is an Open Access article, distributed under the terms of the Creative Commons Attribution licence (http://creativecommons.org/licenses/by/4.0/), which permits unrestricted re-use, distribution, and reproduction in any medium, provided the original work is properly cited. All rights reserved. 0899-823X/2017/3804-0010. DOI: 10.1017/ice.2016.327
} 


\section{MATERIALS AND METHODS}

\section{Setting and Patients}

This study was conducted in a 3,000-bed tertiary referral medical center in Taiwan with $>8,500$ admissions during May 2015. Patients had been treated in the injection room or chemotherapy room at outpatient departments, emergency departments, or hospital wards. All patients who were culture positive for $R$. pickettii from May 3 to June 11, 2015, were eligible for the study. Hospitalwide infection surveillance was conducted. ${ }^{15}$ A case was defined using 2 criteria: (1) a positive $R$. pickettii culture using $\geq 1$ samples of blood or fluid withdrawn via a catheter, and (2) injection-use $20-\mathrm{mL}$ normal saline solution had been used to flush the patient's catheter. This study and report were reviewed and approved, and an exemption was issued by our institutional review board.

\section{Epidemiological Surveillance and Data Collection}

The outbreak period, defined as positive culture for R. pickettii, occurred from May 3 to June 30, 2015. We investigated the associated epidemiological data, including patient data collection, and we conducted field visits and retrieved items with possible contamination. Initially, the first 4 patients were culture positive for $R$. pickettii during May 3-8, 2015. Although these cases were dispersed across 4 wards, the third and the fourth patient specimens positive for these rare bacteria occurred on the same day, and the infection control team immediately launched an investigation. Before May 14, cultures were ordered for 3 additional cases. On May 14, from analysis of epidemiological data, the infection control team highly suspected that injection-use $20-\mathrm{mL}$ normal saline solution was the common source of the infections. Late on May 16, when the laboratory reported 2 positive cultures from unopened $20-\mathrm{mL}$ normal saline ampules of the same lot number (which were suspected glucose-nonfermentative), we immediately stopped using the $20-\mathrm{mL}$ normal saline ampules in all wards. Early on May 17, we confirmed that the bacterial cultures were $R$. pickettii, and we stopped using all $20-\mathrm{mL}$ normal saline ampules throughout the entire hospital. The same day, we notified the Taiwan Centers for Disease Control (Taiwan CDC) and the Taiwan Food and Drug Administration (Taiwan FDA).

During the preoutbreak and outbreak periods, there were no changes in healthcare-associated infection surveillance, culture ordering practices, or intravascular therapy procedures, including intravascular catheter-related care and infusion-related care. We also reviewed laboratory procedures for processing blood cultures and identifying species to exclude the possibility of contamination during the culture process. The blood-culture bottles from clinical units were sent to the laboratory, received barcode scanning, and were placed directly inside the culturing instrument until the culture was complete. This procedure prevented contamination by personnel.
Data on demographic and clinical characteristics, therapeutic devices used, and infusion-related methods were recorded. We also retrieved samples of intravascular catheter- and infusion-related items from patient wards for culture, including unopened $20-\mathrm{mL}$ normal saline ampules and other items (see Tables 1 and 2).

\section{Culture-Positive Patient Discovery and Follow-Up}

We conducted detection and monitoring of patients with possible colonization or infection who might have received the contaminated normal saline from the contaminated pharmaceutical batch between April 10 and the full retrieval of all related products on May 18.

All inpatients who met any of the following criteria were monitored and followed to the last discharged patient (July 20). (1) We followed all patients who had a Port-a-Cath (Bard, Reynosa, Tamaulipas, Mexico) or Hickman catheter (Bard, Reynosa, Tamaulipas, Mexico) implantable venous access device and visited the emergency department. If the patient had fever after the catheter was flushed, then 2 sets of blood cultures were performed and the infectious disease physicians were notified. (2) We followed patients who returned to the injection room as outpatients to have their Port-a-Cath or Hickman catheter flushed. These patients were required to have 3-5-mL fluid withdrawn from the catheter until blood appeared. Microbe cultures were then performed on these samples. (3) We followed all inpatients with Port-a-Caths or Hickman catheters during the outbreak. Physicians were urged to consider suspending the relevant treatment via these implants (ie, before the culture results from the implants were obtained).

\section{Microbiological Identification}

Our microbiology laboratory is certified internationally through the College of American Pathologists. Identification of all R. pickettii isolates was confirmed at the laboratory. The samples of the retrieved items were examined using the Bact/ALERT 3D method (bioMerieux, Durham, NC). Cultures of these retrieved items were processed in an aseptic laboratory room. All R. pickettii clinical isolates from blood cultures and withdrawn fluid from catheter lumens were identified using an automated method with identification cards in the VITEK 2 system and the VITEK-MS system (bioMerieux, Mercy L'Etoil, France). To determine their clonality, all confirmed isolates were subjected to pulsed-field gel electrophoresis (PFGE). The plug preparation and digestion were performed in accordance with a previous study. ${ }^{16}$ Isolates with $>85 \%$ similarity were grouped as the same clone.

\section{RE S U L T S}

\section{Descriptive Epidemiology}

During the outbreak period, 57 culture specimens from 30 case patients were identified as R. pickettii. Blood samples were used 
TAвLE 1. Clinical Characteristics of the Affected Patients for Ralstonia pickettii

\begin{tabular}{lcc}
\hline Variable & $\begin{array}{c}\text { No. of Patients } \\
(n=30)\end{array}$ & $\%^{\mathrm{a}}$ \\
\hline Sex, male & 16 & 53.3 \\
Age, y, mean \pm SD, median (range) & $60.6 \pm 15.0$ & $62(2-83)$ \\
Length of hospital stay, d, mean \pm SD, median (range) & $24.6 \pm 24.2$ & $15(8-109)$ \\
Onset time of symptom, d, mean \pm SD, median (range) & $11.5 \pm 9.9$ & $7(<1-31)$ \\
Main diagnosis & & \\
$\quad$ Neoplasms & 26 & 86.7 \\
$\quad$ Others & 4 & 13.3 \\
Departments & 13 & 43.3 \\
$\quad$ Oncology department & 7 & 23.3 \\
$\quad$ Chest department & 2 & 6.7 \\
Colon and rectal surgery department & 8 & 26.7 \\
$\quad$ Others & 29 & 96.7 \\
Fever & 21 & 70.0 \\
Shaking chills & & \\
\hline
\end{tabular}

NOTE. SD, standard deviation.

${ }^{\mathrm{a}}$ Or median and range, as indicated.

for 24 of these 30 cultures; 6 cultures used fluid withdrawn from Port-a-Caths. Interestingly, 14 of these cultures contained $>2$ specimens. Of these 30 culture-positive $R$. pickettii case patients, 16 were male $(53.3 \%)$. The mean age of the overall cohort was $60.6 \pm 15$ years, and the median hospital stay was 15 days. The most frequent diagnoses were neoplasms (26 cases; 76.7\%). These case patients were admitted to 20 various medical wards in 12 departments, including the oncology department (13 cases; $43.3 \%$ ). Furthermore, 20 case patients $(66.7 \%)$ were undergoing chemotherapy. Overall, 29 case patients (96.7\%) had fever; among them, 21 (72.4\%) had shaking chills and $4(13.8 \%)$ had symptoms within 24 hours (minimum 3 hours) after the catheter flushing. Other case patients presented symptoms up to 31 days after catheter flushing. A single patient died of another cause of disease (Table 1).

These 30 patients had all received intravascular device implants, including 23 patients $(76.7 \%)$ with Port-a-Caths. All case patients had received $20 \mathrm{~mL} 0.9 \%$ normal saline via catheter flushing. Among them, monthly routine Port-a-Cath flushing was performed on 16 patients (53.3\%). Table 2 lists the various reasons that the normal saline solution was used to perform a single catheter flushing.

The first case of BSI due catheter flushing with contaminated normal saline occurred April 29, and the last case occurred on May 16. The dates of catheter flushing that resulted in $R$. pickettii BSI were mostly centralized from May 5 to May 15 (23 cases; 76.7\%). Catheter flushing in case patients occurred in 10 different locations: 2 injection rooms of outpatients, 6 wards, 1 anesthesia induction room, and 1 endoscopy center. The overall incidence of $R$. pickettii in the outpatient injection room was 18 cases $(60 \%)$. For the 30 culture-positive cases, catheter flushing was performed by 24 nurses and 2 anesthesia personnel. t able 2. Clinical Various Devices Used for the Affected Patients

\begin{tabular}{|c|c|c|}
\hline Variable & $\begin{array}{c}\text { No. of } \\
\text { Patients } \\
(n=30)\end{array}$ & $\%$ \\
\hline \multicolumn{3}{|l|}{ Intravascular devices } \\
\hline Port-a-Cath & 23 & 76.7 \\
\hline CVC & 3 & 10.0 \\
\hline Hickman catheter & 2 & 6.7 \\
\hline Peripheral IV catheters (IV lock) & 2 & 6.7 \\
\hline Used $20 \mathrm{~mL}$ normal saline & 30 & 100.0 \\
\hline Used $500 \mathrm{~mL}$ normal saline & 8 & 26.7 \\
\hline Used heparin & 26 & 86.7 \\
\hline Used BD PosiFlush heparin lock flush syringe & 4 & 13.3 \\
\hline \multicolumn{3}{|l|}{ Reason for flushing the catheter } \\
\hline Monthly routine Port-a-Cath flushing & 16 & 53.3 \\
\hline Port-a-Cath flushing after chemotherapy & 6 & 20.0 \\
\hline $\begin{array}{l}\text { Flushing other catheters (Hickman, CVC, IV } \\
\text { lock) }\end{array}$ & 6 & 20.0 \\
\hline Other & 2 & 6.7 \\
\hline \multicolumn{3}{|l|}{ Location of the flushing procedure } \\
\hline General injection rooms of outpatient & 13 & 43.3 \\
\hline Chemotherapy injection rooms of outpatient & 5 & 16.7 \\
\hline Hospital ward & 10 & 33.3 \\
\hline Endoscopy center & 1 & 3.3 \\
\hline Anesthesia induction room & 1 & 3.3 \\
\hline \multicolumn{3}{|l|}{ Site of withdrawal of blood or/and fluid } \\
\hline Peripheral vein & 15 & 50.0 \\
\hline Peripheral vein and Port-a-Cath & 7 & 23.3 \\
\hline Port-a-Cath & 4 & 13.3 \\
\hline Peripheral vein and CVC & 1 & 3.3 \\
\hline CVC & 1 & 3.3 \\
\hline Hickman implant & 1 & 3.3 \\
\hline Other & 1 & 3.3 \\
\hline
\end{tabular}

NOTE. CVC, central venous catheter; IV, intravenous; BD, Becton Dickinson. 
The culture-positive $R$. pickettii specimens were obtained from peripheral veins or/and catheters (18 cases, 60\%). In 9 cases (30\%), positive cultures were obtained during follow-up from peripheral veins, from catheters, withdrawn fluid, or the catheter tip (ie, therapeutic device-related BSI). In 3 cases (10\%), positive cultures were obtained from fluid withdrawn from a Port-a-Cath. In 18 cases, the Port-a-Caths were removed, including cases in which the catheter tip was culture positive ( 9 cases, 50\%), was culture negative (6 cases, $33.3 \%$ ) cases, was culture positive for gram-negative bacilli or gram-positive cocci (2 cases, $11.1 \%$ ), or was not cultured (1 case, $5.6 \%)$.

\section{Microbiological Investigations}

A total of 206 various catheter-related items from units where positive cultures were obtained were cultured. On day 7, bacteria culture confirmed $R$. pickettii in 2 unopened ampules of $20-\mathrm{mL}$ normal saline solution (ie, the same batch but from 2 different wards). The Taiwan CDC sampled and tested 53 ampules of $20-\mathrm{mL}$ normal saline from the same manufactured batch from the pharmaceutical company and also identified the contaminant strain as $R$. pickettii. PFGE indicated that all clinical isolates had similarity of $>90 \%$, confirming the outbreak of the same clone of $R$. pickettii.

\section{I S C USS I O N}

Based on epidemiological data and microbiological and PFGE tests, we determined that the outbreak of $R$. pickettii BSIs occurred via contaminated $20-\mathrm{mL}$ injection-use ampules of normal saline used to flush catheter lumens. Active and prudent epidemiological investigation is very important in detecting potential problems. In our epidemiological investigation, although laboratory testing results had not yet been confirmed, intervention strategies were implemented immediately, including retrieval of items suspected of contamination. We also agreed that a ward or department that yielded $\geq 2$ or more rare species from blood cultures in a short period should be vigilant and should conduct an acuity investigation to determine whether it the contamination was internally or externally derived. ${ }^{5,17}$

\section{Suspicious Items and Plant Investigations}

Several related $R$. pickettii contamination reports have indicated that contaminated sterile $0.9 \%$ saline was the source of outbreaks in 1983 and $1998 .^{2}$ Later, Ryan et al ${ }^{5}$ integrated 55 reports of $R$. pickettii infection distributed in Europe, America, and Asia, including 24 BSI reports (43.6\%). The most common sources of contamination in these outbreaks were distilled water, physiological saline, and catheters. ${ }^{5}$ Recently, Ross et $\mathrm{al}^{9}$ also reported on $R$. pickettii BSI outbreaks caused by contaminated magnesium vials in an intensive care unit. To identify the source of the infections in our outbreak, we obtained the specimens from intravascular catheter- and infusion-related items for microbe culture. Among these retrieved items, we confirmed the same pathogen in 2 unopened 20-mL normal saline ampules in the same supply batch. The results of the PFGE tests were similar, demonstrating that this BSI outbreak event was caused by contamination of normal saline. No further cases occurred after the use of the implicated product was discontinued.

The Taiwan CDC tests for samples from our hospital, Taiwan FDA tests of samples from the pharmaceutical factory, and our hospital laboratory tests revealed that the unopened injectable normal saline ampules were contaminated with R. pickettii. Our hospital tests confirmed the same bacteria in the normal saline ampules from the same batch. This pharmaceutical company used automatic production models and product manufacturing processes for closed systems; however, several laboratory studies have shown that $R$. pickettii can pass through filters, and even low numbers inoculated into $0.9 \%$ sodium chloride solution can proliferate at high temperatures. ${ }^{4,11,13}$ Previous reports pointed out that was the product contaminated by $R$. pickettii may have been caused by either ineffective sterilization or contamination of equipment used in the production process. ${ }^{2}$

\section{Patient Investigations}

Although R. pickettii has low virulence, emergent antibioticresistant opportunistic pathogens, such as $R$. pickettii isolated from hospital environments, could be potentially more dangerous than other more well-known waterborne pathogens. ${ }^{12}$ Bacteremia is the most frequent clinical manifestation of R. pickettii.$^{5,9}$ In particular, this pathogen is more likely to cause infection in patients with impaired immune systems. ${ }^{7,14,17,18}$ Among our 30 cases, the most frequent diagnoses were neoplasms $(86.7 \%)$, and 20 of these patients were undergoing chemotherapy.

In our study, we identified 29 BSI case patients with fever or/and shaking chills; these patients presented with symptoms within 24 hours up to 31 days after the catheter was flushed with the contaminated normal saline. We postulate 2 reasons for this variation in the occurrence of symptoms. First, the contaminated normal saline administered directly through the catheter lumen flashing to the bloodstream resulted in symptoms presenting in a short period of time. Second, in patients undergoing monthly routine catheter flushing, the contaminated normal saline remained in their lumen, allowing R. pickettii colonies to gradually increase over time until they migrated into the bloodstream. Thus, onset of symptoms occurred later. We tested fluid withdrawn from the Port-a-Cath or Hickman catheters of outpatients or inpatients during the outbreak period, and we found 6 culture-positive cases (20\%). We confirmed these speculative culture-positive cases using fluid withdrawn from catheters of asymptomatic patients. $R$. pickettii can grow in various solutions, and our findings are consistent with previous reports. ${ }^{2}$ 
Several studies have illustrated that $R$. pickettii also forms and maintains biofilms inside plastic catheters, making them more resistant to biocides and, consequently, more difficult to eradicate. ${ }^{11,19,20}$ In light of our investigation results, 18 Port-a-Caths were removed, and $50 \%$ of them were culture positive for the catheter tip. Kismet et $\mathrm{al}^{7}$ reported 2 cases of $R$. pickettii bacteremia in patients with Port-a-Caths that could be treated only by removal of the catheters.

In conclusion, during the investigation and retrieval of 6 batches of normal saline ampules, our hospital and the Taiwan FDA confirmed that the R. pickettii were from the same batch and that the other batches were culture negative. We believe that the 20-mL normal saline ampules used for all 30 cases came from the same batch number. Hospital monitoring mechanisms are extremely important in identifying and ending BSI outbreaks such as this one.

\section{ACKNOWLEDGMENTS}

We thank the members of the infection control team for their hard work, and the Department of Nursing and Pharmacy for helping with patients and retrieving test items in Taipei Veterans General Hospital.

Financial support: No financial support was provided relevant to this article.

Potential conflicts of interest: All authors report no conflicts of interest relevant to this article.

Address correspondence to Fu-Der Wang, Division of Infectious Diseases, Department of Medicine, Taipei Veterans General Hospital, No. 201, Section 2, Shih-Pai Road, Taipei, 11217, Taiwan (fdwang@vghtpe.gov.tw).

\section{REFERENCES}

1. O'Grady NP, Alexander M, Burns LA, et al. Guidelines for the prevention of intravascular catheter-related infections. Am J Infect Control 2011;39:S1-S34.

2. Maki DG, Mermel LA. Infections due to infusion therapy. In: Jarvis WR, editor. Bennett and Brachman's Hospital Infection. 5th ed. Philadelphia: Lippincott Williams \& Wilkins; 2007. Pp. 611-647.

3. Yabuuchi E, Kosako Y, Yano I, Hotta H, Nishiuchi Y. Transfer of 2 Burkholderia and an Alcaligenes species to Ralstonia gen-novproposal of Ralstonia pickettii (Ralston, Palleroni and Doudoroff 1973) comb-nov, Ralstonia solanacearum (Smith 1896) comb-nov and Ralstonia eutropha (Davis 1969) comb-nov. Microbiol Immunol 1995;39:897-904.

4. Labarca J, Peterson C, Bendaqa N, et al. Nosocomial Ralstonia pickettii colonization associated with intrinsically contaminated saline solution-Los Angeles, California, 1998. MMWR 1998;47:285-286.

5. Ryan MP, Pembroke JT, Adley CC. Ralstonia pickettii: a persistent gram-negative nosocomial infectious organism. J Hosp Infect 2006;62:278-284.
6. Boutros M, Gonullu N, Casetta A, Guibert M, Ingrand D, Lebrun L. Ralstonia pickettii traced in blood culture bottles. J Clin Microbiol 2002;40:2666-2667.

7. Kismet E, Atay AA, Demirkaya E, et al. Two cases of Ralstonia pickettii bacteremias in a pediatric oncology unit requiring removal of the Port-A-Caths. J Pediat Hematol Onc 2005;27:37-38.

8. Kendirli T, Ciftci E, Ince E, et al. Ralstonia pickettii outbreak associated with contaminated distilled water used for respiratory care in a paediatric intensive care unit. J Hosp Infect 2004;56:77-78.

9. Ross B, Steinmann J, Buer J, et al. Outbreak with Ralstonia pickettii caused by contaminated magnesium vials. Deut Med Wochenschr 2014;139:323-326.

10. Ryan MP, Pembroke JT, Adley CC. Genotypic and phenotypic diversity of Ralstonia pickettii and Ralstonia insidiosa isolates from clinical and environmental sources including high-purity water. Diversity in Ralstonia pickettii. BMC Microbiol 2011;11:194.

11. Adley CC, Saieb FM. Comparison of bioMerieux API 20NE and Remel RapID NF Plus, identification systems of type strains of Ralstonia pickettii. Lett Appl Microbiol 2005;41:136-140.

12. Vincenti S, Quaranta G, De Meo C, et al. Non-fermentative gram-negative bacteria in hospital tap water and water used for haemodialysis and bronchoscope flushing: prevalence and distribution of antibiotic resistant strains. Sci Total Environ 2014;499:47-54.

13. Ryan MP, Adley CC. The antibiotic susceptibility of water-based bacteria Ralstonia pickettii and Ralstonia insidiosa. J Med Microbiol 2013;62:1025-1031.

14. Marroni M, Pasticci MB, Pantosti A, Colozza MA, Stagni G, Tonato M. Outbreak of infusion-related septicemia by Ralstonia pickettii in the oncology department. Tumori 2003;89:575-576.

15. Horan TC, Andrus M, Dudeck MA. CDC/NHSN surveillance definition of health care-associated infection and criteria for specific types of infections in the acute care setting. Am J Infect Control 2008;36:309-332.

16. Labarca JA, Trick WE, Peterson CL, et al. A multistate nosocomial outbreak of Ralstonia pickettii colonization associated with an intrinsically contaminated respiratory care solution. Clin Infect Dis 1999;29:1281-1286.

17. Moreira BM, Leobons MBGP, Pellegrin FLPC, et al. Ralstonia pickettii and Burkholderia cepacia complex bloodstream infections related to infusion of contaminated water for injection. J Hosp Infect 2005;60:51-55.

18. Coenye T, Goris J, De Vos P, Vandamme P, LiPuma JJ. Classification of Ralstonia pickettii-like isolates from the environment and clinical samples as Ralstonia insidiosa sp nov. Int J Syst Evol Microbiol 2003;53:1075-1080.

19. Mijnendonckx K, Provoost A, Ott CM, et al. Characterization of the survival ability of Cupriavidus metallidurans and Ralstonia pickettii from space-related environments. Microb Ecol 2013;65:347-360.

20. Dombrowsky M, Kirschner A, Sommer R. PVC-piping promotes growth of Ralstonia pickettii in dialysis water treatment facilities. Water Sci Technol 2013;68:929-933. 